\title{
Microstructure and mechanical properties of in-situ network composite fibres of PBZT with nylon
}

\author{
C. R. HWANG*, M. F. MALONE, R. J.FARRIS \\ E. L. THOMAS $*$ I \\ Chemical Engineering Department and ${ }^{\ddagger}$ Polymer Science and Engineering Department, \\ University of Massachusetts, Amherst, MA 01003, USA
}

A method for preparing composite fibres by infiltrating nylon into swollen poly ( $p$-phenylene benzobisthiazole) (PBZT) fibre is described. PBZT fibre forms a microfibrillar network structure during the coagulation process. In-situ network composite (IC) fibres may be prepared by exchanging the coagulant with a solution containing the desired matrix material. These new composite fibres exhibit nearly identical mechanical properties and similar thermomechanical properties to those of so-called molecular composite (MC) fibres prepared from isotropic solutions of PBZT and nylon in methane sulphonic acid (MSA). The mechanical properties of these fibres were determined before and after heat treatment under tension. The structure of pure 'PBZT and its composite fibres (ICs' and $\mathrm{MCs}^{\prime}$ ) were characterized using nitrogen adsorption (Brunauer-Emmett-Teller (BET) experiments), small-angle X-ray scattering, and scanning and transmission electron microscopy (SEM and TEM, respectively). The structure of both composite fibres was found to be a microfibrillar network of PBZT in a matrix of amorphous nylon. The average diameters of the PBZT microfibrils were in the range of 10 to $20 \mathrm{~nm}$ for in-situ network composites and approximately $4 \mathrm{~nm}$ for molecular composites.

\section{Introduction}

Isotropic ternary solutions of PBZT, nylon and methane sulphonic acid (MSA) can be spun into a composite fibre by rapid coagulation in water with the intent to form a fine dispersion of the rigid PBZT in a flexible nylon matrix; this process has been studied as a method for the preparation of molecular composites [1]. Coagulating isotropic polymer solution in the form of fibre or film in water resulted in rapid solidification, locking in a fine dispersion of rigid rod macromolecules in the flexible coil matrix [1-4].

However, due to the attractive intermolecular forces between rigid rod PBZT molecules and the large difference in solubilities of PBZT and nylon in the mixture of water and MSA, phase separation into rod-rich and coil-rich phases is rapid and it is difficult to disperse the rodlike molecules on a molecular level in the matrix. A coagulation study indicated that this process is limited by the diffusion of water into the polymer solution [5]. In fact, a continuous PBZT microfibrillar network structure may be formed during the coagulation of PBZT/nylon/MSA solution because of PBZT's greater precipitation rate than nylon. Furthermore, the result of a consolidation study of MC films into larger objects [6] and the tensile properties of the PBZT/nylon MC fibres reported $[7,8]$ indicated that PBZT would form its preferred fibrillar network structure during coagulation from the isotropic composite solutions.

The continuous PBZT structure may provide effective reinforcement for the composite. For example, when nylon was extracted from a $30 / 70 \mathrm{w} / \mathrm{w}$ PBZT/nylon molecular composite fibre using sulphuric acid, the extracted fibre remained intact and actually exhibited higher tensile strength than the original fibre [7]. The existence of a continuous PBZT network is further supported by measurements made after thermally degrading the Nylon 6,6 matrix in the MC fibre to produce a smaller fibre that showed tensile modulus and strength greater than the as-spun fibre [8]. These two studies are consistent with a network of PBZT reinforcing the composite fibre and indicate that the structure of PBZT within the composite fibre supports a significant portion of the load in tension.

It has been shown that coagulated fibres formed during the dry-jet wet-spinning of ordered nematic solutions of PBZT from PBZT/PPA dope consist of a three-dimensional network of microfibrils with diameters in the order of $10 \mathrm{~nm}$ [9-11]. The wet PBZT

\footnotetext{
* Present address: GE Plastics, Selkirk, NY 12158, USA

Present address: Materials Science and Engineering, University of Michigan, Ann Arbor, MI 48109, USA.

TPresent address: Materials Science and Engineering, Massachusetts Institute of Technology, Cambridge, MA, USA.
} 
fibre consists of an interconnected network of oriented microfibrils and micropores in the water-swollen state. The microfibrillar network has appreciable strength even when up to $75 \%$ of its volume is occupied by water; for example, the force required to break a wet PBZT fibre is equal to the force required to break the same fibre after drying [12]. DeTeresa et al. [13] suggested that instead of drying the water from the network, it could be replaced by solid matrix precursor materials, forming a microfibrillar network composite. It was unclear, however, under what conditions it would be feasible to infiltrate a polymer into the PBZT network.

In this paper, we describe the results of infiltrating nylon into a pure PBZT fibre to produce an in-situ network composite (IC) fibre. By comparing the physical properties and morphologies of the IC and MC, the hypothesis for the presence of a continuous PBZT network in the MC fibre will be evaluated.

\section{Experimental procedure}

\subsection{Materials}

The poly ( $p$-phenylene benzobisthiazole), PBZT, was obtained in poly-phosphoric acid (PPA) dope, supplied by SRI International, and had a weight-average $M_{\mathrm{w}}$ of 38000 corresponding to an aspect ratio of about 380 . The PPA in the dope was removed to obtain pure PBZT flakes. The amorphous nylon used was Du Pont Zyte ${ }^{\circledR} 330\left(T_{\mathrm{g}}=130^{\circ} \mathrm{C}\right)$, a copolymer of aliphatic and aromatic polyamide. The solvent was 98\% methane sulphonic acid, MSA, used as received from Aldrich Chemical Company.

\subsection{Fibre Preparation}

Fibres were spun through a die with a single $330 \mu \mathrm{m}$ diameter hole into a water bath without an air-gap at approximately $20^{\circ} \mathrm{C}$. The details of the spinning procedure were described elsewhere [7]. PBZT fibre was also spun from a $2 \%$ isotropic solution in MSA and kept under water to avoid the structural collapse of the wet fibre that occurs during the drying process [12]. The water was then replaced with $95 \%$ sulphuric acid by soaking the wet fibre in an acid bath.

Three batches of such PBZT fibres, filled with sulphuric acid, were then transferred into nylon/sulphuric acid solutions containing 0,5 , and $20 \mathrm{wt} \%$ polymer. An elemental analysis of sulphur and nitrogen in the fibres indicated that a maximum nylon content was achieved after two days of infiltration. After two weeks of infiltration, the fibres were recoagulated in a water bath and dried in air on take-up wheels. The excess nylon on the surface of the composite fibres from the re-coagulation process was removed after the air-drying process. The samples were designated as IC-0, IC-5 and IC-20, corresponding to the nylon concentrations. These fibres are referred to as in-situ network infiltrated composites, since the pure PBZT microfibrillar network was present prior to the formation of the composites. Based on the increase of the diameter of IC-5 fibre and on an elemental analysis, the weight ratio of PBZT to nylon was found to be nearly unity (1.1). The IC-20 fibre had a PBZT/nylon weight ratio of 0.37 .

Molecular composite fibres of PBZT/nylon were also prepared as described elsewhere [7]. PBZT/nylon fibres with a polymer ratio of $25 / 75$ and $50 / 50 \mathrm{w} / \mathrm{w}$ were spun from near critical concentrations (4.0 and $3.4 \mathrm{wt} \%$ solutions), and designated as $25 / 75$ and $50 / 50 \mathrm{MC}$ (as-spun). The orientation of the fibres was induced by heat-treatment under tension (HTT).

A dynamometer, calibrated with dead weights, was used to control the tension of the fibre during heattreatment. Fibres were heat-treated at various temperatures $\left(190^{\circ} \mathrm{C}, 300^{\circ} \mathrm{C}, 380^{\circ} \mathrm{C}\right)$ under a nitrogen atmosphere and at a tension of 20 to $60 \mathrm{MPa}$ depending on the composition of PBZT. The oven residence time for heat-treatment was $2 \mathrm{~min}$.

\subsection{Characterization of fibres}

\subsubsection{Mechanical properties}

Diameters of the fibres were measured with an optical microscope under $100 \times$ or $400 \times$ magnification. The tensile properties were measured using an Instron Universal Testing Machine at a crosshead speed of $1 \mathrm{~mm} \mathrm{~min}^{-1}$, under ambient conditions. Samples were mounted on paper tabs with Devcon 5 min epoxy and then cured overnight. The shear moduli were obtained from free torsional oscillatory experiments described elsewhere [14].

The compressive strengths were measured from a recoil test developed by Allen [15] with slight modifications. Instead of stressing the fibre in an Instron with both ends fixed, the fibre was stressed by hanging a dead weight to a desired tensile stress on one end of the fibre in order to reduce any extra stresses induced by cutting the fibre. The gauge length of all the fibres tested was $2 \mathrm{~cm}$. The cutting was performed with sharp surgical scissors at the centre of the gauge length. The load cell was connected to a Nicolet Digital Oscilloscope to monitor any extra stresses induced by cutting the fibre. After performing a number of tensile recoil tests, the threshold stress value just sufficient to produce snap-back compressive damage was identified from a series of observations through an optical micrscope.

The length change of the fibres was measured as a function of temperature $\left(50^{\circ} \mathrm{C}\right.$ to $300^{\circ} \mathrm{C}$, under a dry nitrogen atmosphere) by a thermomechanical analyser (Perkin Elmer TMA-2) with a tension of approximately $5 \mathrm{MPa}$. The heating rate was $10^{\circ} \mathrm{Cmin}^{-1}$.

\subsubsection{Morphology}

The porous fibrillar structure of the wet fibre undergoes a dramatic collapse during air-drying. However, the microstructure of the as-spun fibres can be partially preserved by freeze-drying. The wet fibre samples were quenched in liquid nitrogen and then freeze dried by vacuum sublimation of water. The freezedrying process required 24 to $48 \mathrm{~h}$. Surfaces of some of the freeze-dried fibres prepared by the peelback method [16] were observed with a JEOL 35-CF SEM where the internal morphological features could be 
TABLE I Composition, diameter and mechanical properties of the as-spun fibres; $G$ : shear modulus, $E$ : tensile modulus, $\sigma_{t}$ : tensile strength, $\varepsilon_{\mathrm{r}}$ : strain at break. \pm represents the range of deviation from the average from at least six samples.

\begin{tabular}{llccccc}
\hline Fibre & PBZT w/w & $D(\mu \mathrm{m})$ & $G(\mathrm{GPa})$ & $E(\mathrm{GPa})$ & $\sigma_{\mathrm{t}}(\mathrm{MPa})$ & $\varepsilon_{\mathrm{t}}(\%)$ \\
\hline IC-0 & 1.00 & $65 \pm 3$ & $1.5 \pm .1$ & $13.2 \pm .6$ & $340 \pm 20$ & $40 \pm 3$ \\
PBZT-AS & 1.00 & $67 \pm 3$ & $1.5 \pm .1$ & $13.0 \pm .5$ & $340 \pm 20$ & $42 \pm 3$ \\
IC-5 & 0.52 & $91 \pm 4$ & $1.4 \pm .1$ & $8.0 \pm .5$ & $220 \pm 20$ & $50 \pm 10$ \\
$50 / 50 \mathrm{MC}$ & 0.50 & $85 \pm 3$ & $1.4 \pm .1$ & $8.0 \pm .5$ & $200 \pm 25$ & $45 \pm 6$ \\
IC-20 & 0.27 & $110 \pm 4$ & - & $4.1 \pm .5$ & $180 \pm 10$ & $50 \pm 5$ \\
$25 / 75 \mathrm{MC}$ & 0.25 & $108 \pm 5$ & - & $4.5 \pm .5$ & $95 \pm 10$ & $15 \pm 5$ \\
\hline
\end{tabular}

TABLE II Diameter and mechanical properties of the heat-treated fibre; $\sigma_{c}$ is the compressive strength.

\begin{tabular}{lcccccc}
\hline & $D(\mu \mathrm{m})$ & $G(\mathrm{GPa})$ & $E(\mathrm{GPa})$ & $\sigma_{\mathrm{t}}(\mathrm{MPa})$ & $\sigma_{\mathrm{c}}(\mathrm{MPa})$ & $\varepsilon_{\mathrm{t}}(\%)$ \\
\hline PBZT-HT $^{\mathrm{a}}$ & $49 \pm 1$ & $2.0 \pm 1$ & $80 \pm 5$ & $560 \pm 30$ & $240 \pm 20$ & $0.8 \pm .1$ \\
IC-5HT $^{\mathrm{b}}$ & $73 \pm 1$ & $1.8 \pm .1$ & $43 \pm 3$ & $430 \pm 30$ & $270 \pm 20$ & $1.4 \pm .1$ \\
$50 / 50 \mathrm{MC}-H T^{\mathrm{c}}$ & $72 \pm 1$ & - & $44 \pm 2$ & $430 \pm 30$ & - & $1.8 \pm .1$ \\
$50 / 50 \mathrm{MC}^{\mathrm{H}} \mathrm{HT}$ & $70 \pm 1$ & $1.7 \pm .1$ & $48 \pm 2$ & $460 \pm 30$ & $245 \pm 20$ & $1.1 \pm .1$ \\
$50 / 50 \mathrm{MC}^{\mathrm{b}} \mathrm{HT}^{\mathrm{a}}$ & $69 \pm 1$ & - & $49 \pm 2$ & $440 \pm 30$ & - & $1.0 \pm .1$ \\
\hline
\end{tabular}

(heat-treatment temperature $={ }^{\mathrm{a}}\left(380^{\circ} \mathrm{C}\right),{ }^{\mathrm{b}}\left(300^{\circ} \mathrm{C}\right),{ }^{\mathrm{c}}\left(190^{\circ} \mathrm{C}\right)$.

imaged. The samples were sputter-coated with $30 \mathrm{~nm}$ of gold to avoid charging and electron beam damage.

Nitrogen adsorption was used to determine the internal surface area of the freeze-dried fibres. This technique is widely used for catalyst characterization in the petroleum industry and is described in detail by Brunauer [17].

TEM was also employed to study the microstructure of the composite fibres. Composite fibres were embedded in Spurr epoxy resin and sectioned with a diamond knife in a Sorvall room temperature microtome at an angle of 50 degrees to the fibre axis. The sample thickness was approximately $50 \mathrm{~nm}$. Both bright field and dark field images were obtained with a JEOL 2000 FX TEM operating at $200 \mathrm{kV}$.

Small-angle X-ray scattering (SAXS) patterns were obtained for both $50 / 50 \mathrm{MC}$ and IC-5 fibre bundles using a Kratky camera with the slit-collimated incident beam parallel to the fibre axis. This technique has been proved useful to characterize the size scale for phase separation in polymer composites [18].

\section{Results and discussion}

\subsection{Mechanical properties}

The mechanical properties of the as-spun and heattreated composite fibres (both IC and MC) are shown in Tables I and II. The tensile modulus of PBZT/nylon fibres can be increased by a factor of 5 to 6 and tensile strength by a factor of 2 as a result of heat-treatment under tension. The tensile properties are not a strong function of the heat-treatment temperatures studied here. The IC-0 fibre exhibits tensile properties similar to those of the as-spun PBZT fibre. This indicates that the $95 \%$ sulphuric acid does not affect the tensile properties of $2 \%$ as-spun PBZT fibre. The as-spun and heat-treated IC-5 fibres exhibited mechanical properties indistinguishable from those of $50 / 50 \mathrm{MC}$ fibres of the same composition prepared from isotropic solutions. The shear moduli and compressive strength of the PBZT fibre were not improved by the blending or infiltration of nylon into the fibre. The IC-20 fibre exhibited tensile strength and strain at a superior break to the $25 / 75 \mathrm{MC}$ fibres despite the fact that both fibres had the same tensile moduli.

The fact that the strengths and strains at break for the 50/50 MC and IC-5 composites are nearly identical is strong evidence for the presence of a continuous PBZT network in the MC fibre. The superior strength and strain at break of the IC-20 fibre compared to the $25 / 75 \mathrm{MC}$ can be explained as a result of the formation of a more perfect PBZT network in the IC case since there is more nylon present to disrupt the continuity of the PBZT network formation during the coagulation of $25 / 75 \mathrm{MC}$ solution.

The strain-temperature $(\varepsilon-T)$ behaviour of the $50 / 50 \mathrm{MC}, \mathrm{IC}-5$ and the pure PBZT fibres are shown in Fig. 1. The as-spun fibres exhibit thermal hysteresis, but after heating, the subsequent cooling and reheating cycle displays reversible $\varepsilon-T$ behaviour. The data shows that after heat-treatment under tension the fibres all exhibit the same $\varepsilon-T$ slope, which is the same as the heat-treated PBZT fibres. The fibre lengths are stabilized by heat-treatment.

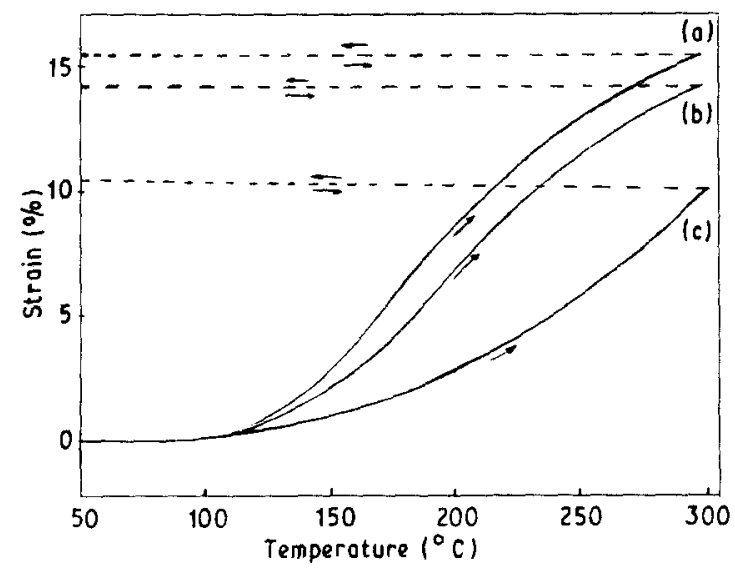

Figure 1 TMA scans of (a) 50/50 MC, (b) IC-5 and (c) pure PBZT fibres. The subsequent cooling and re-heating are in dashed lines. 


\subsection{Morphology}

Wide angle X-Ray scattering patterns of similar MC and IC fibres spun from isotropic solutions show only a very slight orientation of PBZT in the as-spun condition $[18,19]$. After heat treatment, the PBZT network becomes oriented, but the nylon rings remain nearly isotropic $[18,19]$.

The peeled surface of the freeze-dried PBZT fibre appears fibrillar and uniform, as shown by SEM in Fig. 2. The morphology of the fracture surface in the centre of the fibre does not appear different from areas near the edge. This is evidence that there is not a pronounced skin-core effect in the fibre. More detailed interpretation of SEM data is hindered by limited resolution and possible surface artefacts during fracture of the sample.

The physical adsorption of gases onto solid surfaces can be used to determine the surface area if the area of the adsorbing molecule and the amount of molecules necessary to cover the surface completely (with a monolayer) are known [9]. The diameter of the wet PBZT fibre spun from a $2 \mathrm{wt} \%$ solution is $330 \mu \mathrm{m}$. The largest freeze-dried fibre diameter was $230 \mu \mathrm{m}$, whereas the diameter of a fibre dried in air at a constant length was $65 \mu \mathrm{m}$. From the nitrogen adsorption (BET experiments) data, the surface area of the freeze-dried fibre measured was $275 \mathrm{~m}^{2} \mathrm{~g}^{-1}$ and the outside surface of the air-dried fibres was less than $0.1 \mathrm{~m}^{2} \mathrm{~g}^{-1}$. In fact, the measured specific surface area of the freeze-dried sample is higher than that of the average catalysts $\left(150 \mathrm{~m}^{2} \mathrm{~g}^{-1}\right)$. To interpret the measured quantity surface area $(A)$ a cylindrical fibrillar model of the microstructure for PBZT was assumed. The average diameter $(D)$ of the fibrils can then be expressed as $D=4 /\left(A^{*} \rho\right)$, where $\rho$ is the density of the fibrils $\left(1.5 \mathrm{~g} \mathrm{cc}^{-1}\right)$. The average diameter of PBZT microfibrils was determined to be $10 \mathrm{~nm}$. The calculated diameter of the PBZT microfibrils is nearly identical with the previous value determined by TEM and SAXS $[10,11]$.

Fig. 3a-c show dark field images of the IC-5, $50 / 50 \mathrm{MC}$, and 50/50 MC-HTT fibres. The field of view in each image shows a region of the sample near the epoxy embedding medium. The epoxy is seen as a uniform region at the upper right in Fig. $3 \mathrm{a}$ and $\mathrm{b}$ and

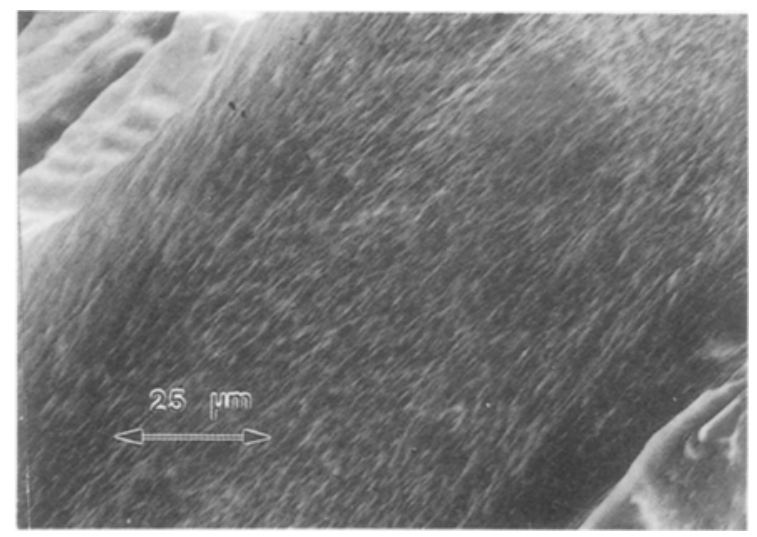

Figure 2 Scanning electron micrographs of the peeled surface of a freeze-dried PBZT fibre spun from a 2 wt $\%$ solution. at the upper left in Fig. 3c. The dark lines running from top to bottom in each image are tears due to imperfections in the diamond microtome knife. The PBZT has a higher electron density than nylon and therefore appears brighter by comparison in dark field imaging.

Fig. 3a shows clearly the interconnected network of PBZT which appears as a light, connected cellular structure within the body of the fibre. The appearance of the network is remarkably uniform over the entire fibre. The size of individual microfibrils is in the order of $10-20 \mathrm{~nm}$ and the nylon-rich cells between microfibrils are about $80 \mathrm{~nm}$ in diameter. The three-dimensionally interconnected nature of the PBZT network was confirmed by visualizing stereo pairs of images taken with 6 degrees of tilt between them. It is interesting to compare the isotropic appearance of the network seen in this work (by coagulating an isotropic solution) with the anisotropic appearance of the PBZT network formed by coagulation of a nematic solution [11].

Careful observation of the outer $1 \mu \mathrm{m}$ of the fibre (Fig. 3a) shows that the PBZT network is of slightly finer scale in this region. In this area the spacing between microfibrils appears to be less than $40 \mathrm{~nm}$. These TEM results show that the IC-5 consists of a continuous composite of a PBZT microfibrillar network with nylon uniformly precipitated onto the network throughout the cross-section of the fibre. The $10-20 \mathrm{~nm}$ diameter of the microfibrillar network is consistent with the nitrogen adsorption experiments and prior SAXS measurements [11].

The dark field image of the 50/50 MC fibre shown in Fig. $3 b$ appears much more uniform than the IC fibre shown in Fig. 3a. However, close inspection and comparison with the uniform epoxy resin shows fluctuations in image intensity indicating that some amount of phase separation has taken place. Evidently, the scale of phase separation in the MC 50/50 is of the order or smaller than the section thickness $(50 \mathrm{~nm})$, causing the projected structure to appear approximately uniform. Nevertheless, the degree of phase separation is large enough to cause significantly more contrast variation than in the epoxy matrix. Hence, we estimate the scale of phase separation to be in the order of 3-5 $\mathrm{nm}$. The presence of a weak skincore effect is again apparent as a slightly more homogeneous outer region ( 0.5 to $1 \mu \mathrm{m})$ of the fibre.

The morphology of the MC 50/50 fibre was also investigated after heat-treatment at $380^{\circ} \mathrm{C}$ under a stress of $40 \mathrm{MPa}$. As the dark field TEM in Fig. 3c shows, the inside of the 50/50 MC-HTT fibre appears to have a uniform morphology similar to the $50 / 50 \mathrm{MC}$ fibre. The phase separation in the heattreated sample appears slightly more coarse than the as-spun sample (Fig. 3b) but still much less well defined than the IC-5 fibre (Fig. 3a). Apparently the microfibrillar network in the MC fibres is thermally stable; the heat-treatment under tension does not significantly enlarge either the fibrillar size or the spacing between the fibrils.

Fig. $3 \mathrm{~d}$ is a bright field micrograph of the MC 50/50-HTT fibre showing a region where blobs 

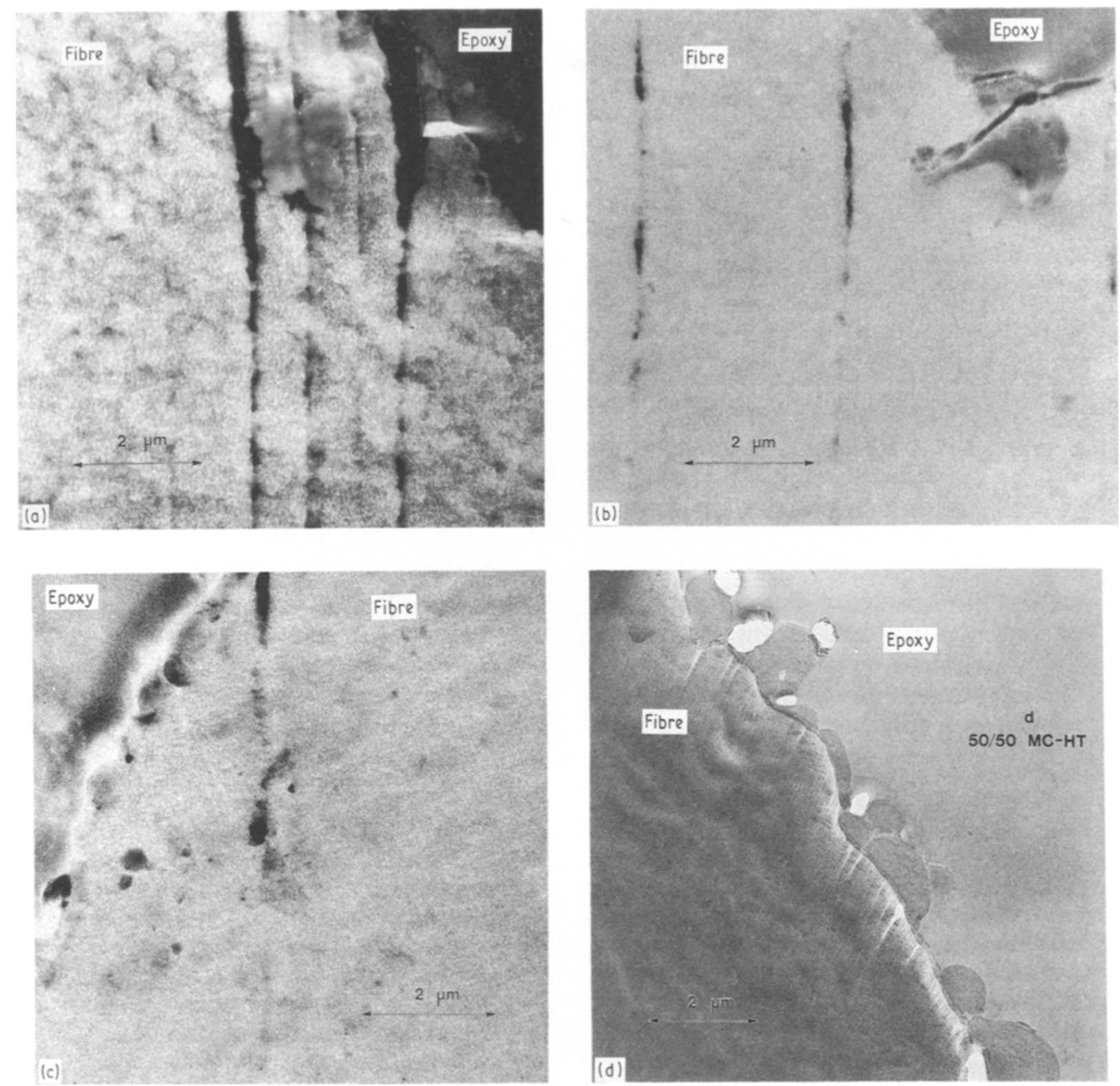

Figure 3 TEM dark field images of (a) IC-5, (b) 50/50 MC, (c) 50/50 MC-HT fibres and (d) TEM bright field image of 50/50 MC-HT fibre showing 'blobs' of nylon exuded at the surface.

with uniform electron density can be seen on the surface of the sample. In the bright field image PBZT is darker than the nylon. The blobs are indicative of a homogeneous phase being exuded out from the bulk of the fibre during heat treatment. The mass thickness contrast of these blobs is intermediate between that of the epoxy resin and the MC fibre, suggesting their composition to be nylon.

Fig. 4a and $\mathrm{b}$ show SEM images of the MC 50/50 fibre before and after heat-treatment. The blobs of exuded material can now be clearly identified on the surface of the MC 50/50-HTT fibre. These blobs could be removed by washing the sample in sulphuric acid, confirming their composition as nylon-rich.

The thermal stability of the PBZT network seen in these morphological studies is corroborated by the fact that the 50/50 MC-HTT sample did not flow as might be expected for a true molecular composite of PBZT in nylon.

Chuah et al. [4] investigated the kinetics of phase separation in PBZT/Nylon 6,6 MCs by backscattered SEM and small-angle light scattering (SALS). They found evidence for large $(2-7 \mu \mathrm{m})$ phase separated domains when heat treating MC films prepared from isotropic solution. Here, we found that the entire interior of the MC 50/50-HTT fibre remained in the form of a fine-scale microfibrillar network with no indication of large phase separated domains. Our results are consistent with a uniform syneresis of the PBZT network during heat-treatment accompanied by the formation of large, nylon blobs at the surface of the sample.

SAXS was used to probe further the morphology of the samples in the $5-100 \mathrm{~nm}$ size range. $I(S)$ is the smeared intensity due to the line slit-collimator where $S$ is the scattering vector $4 \pi / \lambda \sin \theta$. A plot of $\log$ $[I(S)]$ versus $S$ for IC-5 and $50 / 50 \mathrm{MC}$ is shown in Fig. 5. From these data, the characteristic dimension of the microstructure can be estimated if a model for the geometry is assumed. 

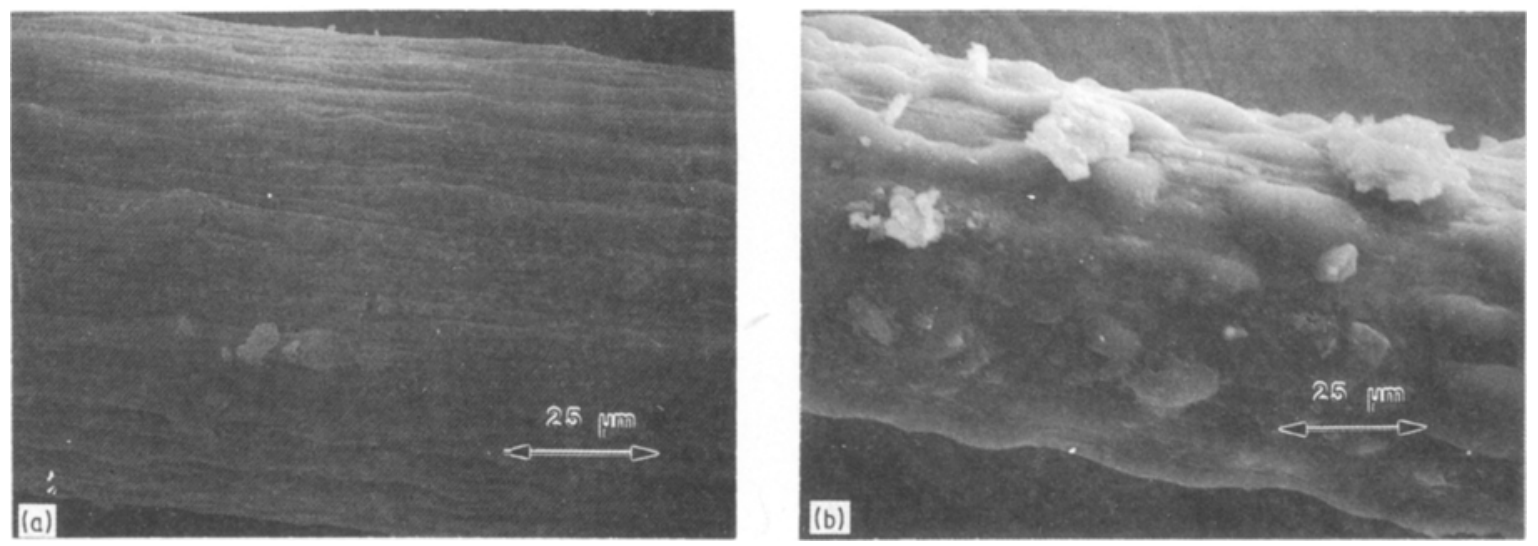

Figure 4 Scanning electron micrographs of a $50 / 50 \mathrm{PBZT} /$ Zytel $^{\oplus} 330$ molecular composite fibre spun from a 3 wt $\%$ polymer solution (a) AS, (b) HTT at $380^{\circ} \mathrm{C}$.

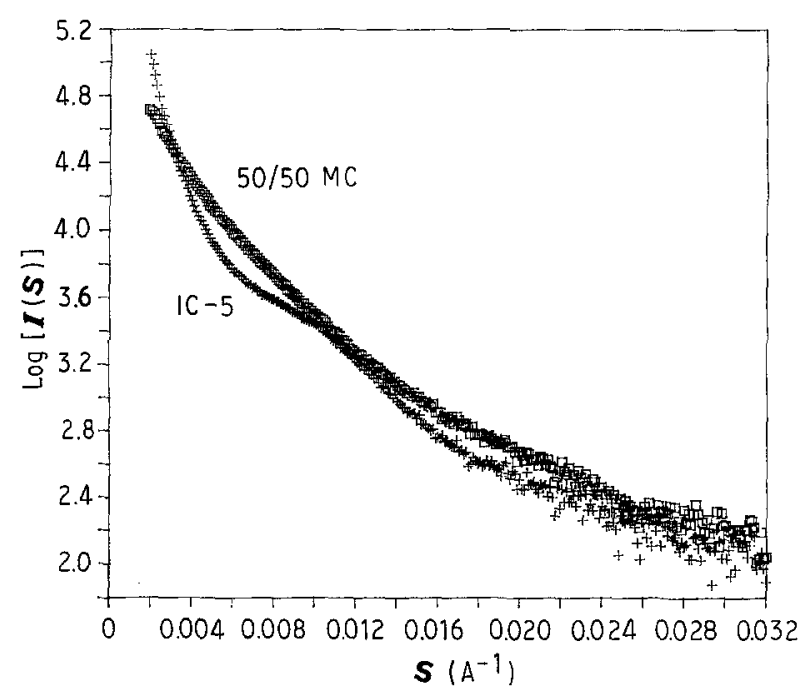

Figure 5 SAXS patterns of IC-5 and 50/50 MC fibres using a slitcollimated incident beam.

The monotonic fall off of the scattered intensity with scattering angle suggests a random two phase system. Debye's random two-phase model states that the smeared scattered intensity, $I(S)$, and the correlation length for the two phase material, $l_{\mathrm{c}}$, can be related by $[I(S)]^{-2 / 3}=\left(1+4 \pi^{2} l_{\mathrm{c}}^{2} S^{2}\right) / A^{\prime}[11,18]$ where $A^{\prime}=A / 2 l c$ and $A$ is a scattering constant. The quantity $[I(S)]^{-2 / 3}$ should be a linear function of $S^{2}$. The correlation length of the microstructure in $50 / 50 \mathrm{MC}$ fibre obtained by this model is approximately $4 \mathrm{~nm}$ as can be deduced from Fig. 6 .

These SAXS results are consistent with the TEM results. The thickness of the sample is one order of magnitude greater than the characteristic dimension of the random two phase for the $\mathrm{MC}$ fibre determined by SAXS. Evidently, the random two-phase model does not fit the scattering data for the IC-5 as well as the MC 50/50 data because the microfibrillar structure is more defined and regularly-shaped in IC-5 fibre, as shown by TEM in Fig. 3b.

\section{Conclusion}

A novel method of infiltrating nylon into a pure PBZT network was developed to produce an in-situ composite (IC) fibre. The mechanical properties and mor-

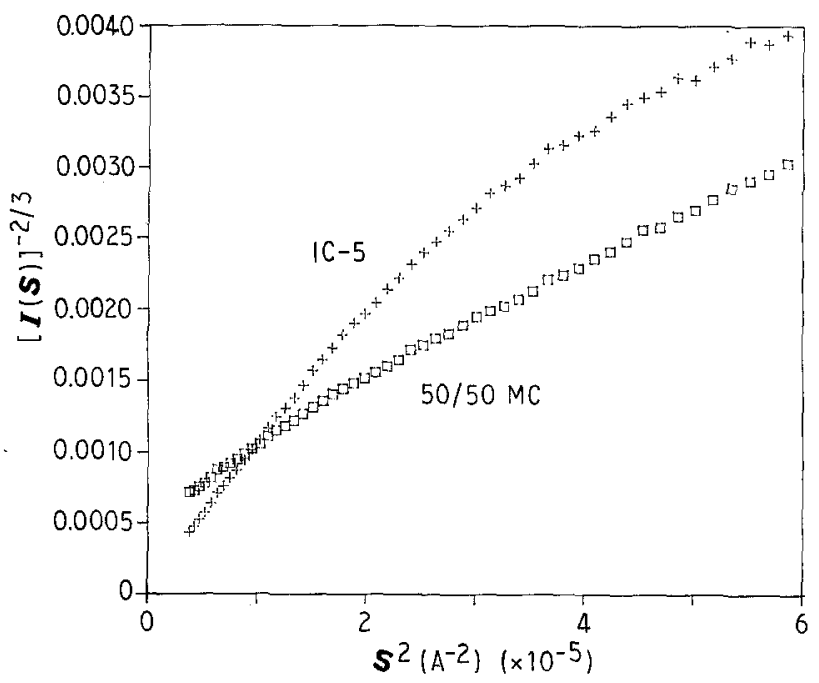

Figure 6 SAXS data plotted to fit Debye's random two-phase model.

phology of the IC and molecular composite (MC) fibres were examined and compared. The tensile properties of the IC fibre were found to be as good as or better than an MC fibre depending on the composition of PBZT and nylon. Since PBZT forms a continuous network phase in the IC fibre, these results are strong evidence for the presence of a continuous PBZT network in the MC fibre. The size scale of the microfibrillar network in IC $(10$ to $20 \mathrm{~nm}$ in diameter) is three to five times greater than that of MC's (roughly $4 \mathrm{~nm}$ in diameter). The PBZT network structure in the MC is stable during heat treatment, although syneresis occurs causing nylon blobs to be exuded at the surface. These results indicate that it is not necessary to achieve molecular level dispersion in order to achieve good mechanical properties.

\section{Acknowledgements}

This research was support by AFWAL/MLBP Contract No. F-33615-83-K-5001 and E.I. Du Pont de Nemours, Inc. We thank D. Gobran of UMass for help in obtaining the SAXS data and C. Shambelan, G. M. Prilutski and W. C. Uy of Du Pont for providing some of the materials. 


\section{References}

1. W.-F. HWANG, D. R. WIFF, C. L. BENNER and T. E HELMINIAK, J. Macromol. Sci.-Phys. B22 (1983) 231.

2. W.-F. HWANG, D. R. WIFF, C. VERSCHOORE, G. E. PRICE, T. E. HELMINIAK and W. W. ADAMS, Polym. Engng. Sci. 23 (1983) 789.

3. W.-F. HWANG, D. R. WIFF, T. E. HELMINIAK and W W. ADA MS, ACS Div. Org. Coat. Plast. Chem. Preps. $48(1982)$ 929.

4. H. H. CHUAH, T. KYU and T. E. HElminiak, Polym. Mater. Sci. Engng. 56 (1987) 58.

5. C. R. HWANG, M. F. MALONE and R. J. FARRIS, J. Mater. Sci. 26 (1991) 1762.

6. Idem., Paper Number C02.30, Conference on Emerging Technologies in Materials (AIChE, Minnesota, 1987).

7. S. M. WICKLIFFE, M. F. MALONE and R. J. FARRIS, J. Appl. Polym. Sci. 34 (1987) 931

8. C. A. GABRIEL, PhD thesis, University of Massachusetts, Amherst (1987).

9. Y. COHEN and E. L. THOMAS, Polym. Engng. Sci. 25 (1985) 1093
10. Idem., Macromol. 21 (1988) 433.

11. Idem., ibid. 21 (1988) 436.

12. L. A. POTTICK and R. J. FARRIS, TAPPI Proceedings: 1985 Non Woven Symposium 85 (1985) 65.

13. S. J. DeTERESA, Y. COHEN and R. J. FARR IS, UK Patent, GB 2195672 A (1988).

14. S. R. ALLEN, PhD thesis, University of Massachusetts, Amherst (1983).

15. Idem., J. Mater. Sci. 22 (1987) 853.

16. L. C. SAWYER and D. T. GRUB B, in "Polymer Microscopy" (Chapman and Hall, New York, 1987) p. 85.

17. S. BRUNAUER, in "The Adsorption of gases and vapors" (Princeton University Press, Princeton, N.J., 1945) p. 41

18. O. A. NEHME, C. A. GABRIEL, R. J. FARRIS, E. L. THOMAS and M. F. MALONE, J. Appl. Polym. Sci. 35 (1988) 1965.

19. C. R. HWANG, PhD Dissertation, The University of Massachusetts at Amherst (1989).

Received 24 July

and accepted 22 November 1989 